\title{
Soil moisture retrieval from multi-instrument observations: Information content analysis and retrieval methodology
}

\author{
J. Kolassa, ${ }^{1}$ F. Aires, ${ }^{1,3}$ J. Polcher, ${ }^{2}$ C. Prigent, ${ }^{3}$ C. Jimenez, ${ }^{3}$ and J. M. Pereira ${ }^{4}$ \\ Received 24 May 2012; revised 16 November 2012; accepted 20 November 2012; published 17 May 2013.
}

[1] An algorithm has been developed that employs neural network technology to retrieve soil moisture from multi-wavelength satellite observations (active/passive microwave, infrared, and visible). This represents the first step in the development of a methodology aiming to combine beneficial aspects of existing retrieval schemes. Several quality metrics have been developed to assess the performance of a retrieval product on different spatial and temporal scales. Additionally, an innovative approach to estimate the retrieval uncertainty has been proposed. An information content analysis of different satellite observations showed that active microwave observations are best suited to capture the soil moisture temporal variability, while the amplitude of the surface temperature diurnal cycle is best suited to capture the spatial variability. In a synergy analysis, it has been found that through the combination of all observations the retrieval uncertainty could be reduced by $13 \%$. Furthermore, it was found that synergy benefits are significantly larger using a data fusion approach compared to an a posteriori combination of retrieval products, supporting the combination of different retrieval methodology aspects in a single algorithm. In a comparison with model data, it was found that the proposed methodology also shows potential to be used for the evaluation of modeled soil moisture. A comparison with in situ observations showed that the algorithm is well able to capture soil moisture spatial variabilities. It was concluded that the temporal performance can be improved through incorporation of other existing retrieval approaches.

Citation: Kolassa, J., F. Aires, J. Polcher, C. Prigent, C. Jimenez, and J. M. Pereira (2013), Soil moisture retrieval from multi-instrument observations: Information content analysis and retrieval methodology, J. Geophys. Res. Atmos., 118, 4847-4859, doi:10.1029/2012JD018150.

\section{Introduction}

[2] Compared to its other components, surface soil moisture constitutes only a very small part of the global hydrological cycle (about 0.005\%) [Wagner, 1998]. Nevertheless, it is of great importance for the development of land surface and climatological models and to observe trends in the surface water budget. This is because the moisture content of the soil strongly influences the interaction between the land surface and the atmosphere through its effect on soil evaporation and transpiration. In addition, soil moisture determines the distribution of precipitation into infiltration and surface runoff and controls plant growth. Hence, there is a strong need for a global and long-term soil moisture

\footnotetext{
${ }^{1}$ Estellus, S.A.S, Paris, France.

${ }^{2}$ Laboratoire d'Etude du Rayonnement et de la Matière en Astrophysique, CNRS, Paris, France.

${ }^{3}$ Laboratoire de Météorologie Dynamique, CNRS, Paris, France.

${ }^{4}$ Instituto Superior de Agronomia, Universidade Tecnica de Lisboa, Lisbon, Portugal.

Corresponding author: J. Kolassa, Estellus S.A.S., 93 Boulevard de Sébastopol, F-75014, Paris, France. (jana.kolassa@estellus.fr)

(C)2012. American Geophysical Union. All Rights Reserved. 2169-897X/13/2012JD018150
}

estimate. While in situ measurements of soil moisture still offer the most important estimates, the network of measurement stations is sparse, limited to a few regions, and restricted in temporal resolution and duration. Furthermore, since in situ measurements are only representative of a single point, the scaling of these measurements to model or satellite scales introduces a high degree of uncertainty in the intercomparison. Hence, at this point the only reasonable option for the development of a global, long-term soil moisture product is through retrieval from satellite observations. Generally, satellite observations are sensitive to the upper soil layer and as such are used to retrieve surface soil moisture. The Soil Moisture and Ocean Salinity (SMOS) Mission [Kerr et al., 2010] launched in 2009 represents the first satellite sensor dedicated to the retrieval of soil moisture. It carries an $L$ band radiometer and employs an interferometric approach in order to distinguish the soil moisture signal from other parameters affecting the measured brightness temperature, such as for instance the vegetation cover or the soil roughness. SMOS is soon to be joined by the Soil Moisture Active Passive (SMAP) Mission [Entekhabi et al., 2010], which aims to exploit the synergy of active and passive microwave observations. Both missions present the state-of-the-art in soil moisture retrieval, however, only few 
years of SMOS data are available at this point, which limits its application in climatological studies.

[3] Thus in order to create a long-term, global soil moisture product, one is obliged to perform a retrieval from older instruments not dedicated to measuring soil moisture. A large amount of work in this direction has already been performed resulting in a number of different satellite retrieval products. Owe et al. [2001] and Njoku et al. [2003] both perform an inversion of different microwave emission models to retrieve soil moisture from Advanced Microwave Scanning Radiometer-EOS brightness temperatures, while Wagner [1998] applied a change detection algorithm to active microwave observations from ERS and MetOp-A. Furthermore, Owe et al. [2008] extended their retrieval to other passive microwave sensors and combined the resulting estimates in order to create a 30 year soil moisture database. Additionally, Liu et al. [2011] merged soil moisture retrievals from active and passive microwave sensors to exploit their complementarity and generate a long-term soil moisture database. Prigent et al. [2005] developed a retrieval scheme based on neural networks and studied the sensitivity of different satellite observations to soil moisture. Despite all these efforts, soil moisture retrieval from non-dedicated sensors still remains a great challenge, and each of the retrieval schemes presented above is subject to limitations (often in densely vegetated or arid regions).

[4] The purpose of this paper is to present the first steps in the development of a methodology that aims to combine the best aspects of the existing algorithms and thus improve the quality of soil moisture retrievals from non-dedicated sensors. The methodology proposed represents a multiwavelength retrieval, which combines not only passive and active microwave observations, but additionally infrared and visible data. This aims on one hand to account for possible contaminations of the microwave data, for example, through the presence of vegetation or surface temperature effects. More importantly, however, it is presumed that the addition of these observations will contribute information on the soil moisture variability, which is not captured by the microwave observations. The proposed algorithm utilizes neural network technology, which facilitates data fusion of different satellite observations [F. Aires et al., 2012]. As such the proposed methodology represents a statistical method different from the inversion of microwave emission models or the regression algorithm other retrievals are based on. This means that the algorithm will not encounter the same complications as other techniques and the retrieved soil moisture thus represents an alternative product, which could complement existing retrieval products. While at this point the neural network is calibrated on a model, the plan is to eventually use SMOS retrievals for the calibration to obtain a purely observation-dependent product compatible with the SMOS data. Additional steps aiming to incorporate existing soil moisture retrieval expertise in the proposed neural network algorithm will also be discussed.

[5] The purpose of this paper is to present the retrieval methodology that has been developed and several analyses that were performed as part of the development and a first evaluation of the preliminary retrieval product against different sources of soil moisture data. The data sets used in the analysis and the processing applied to them is presented in section 2. Section 3 discusses several aspects of the retrieval methodology proposed here, including the retrieval algorithm, the quality metrics developed to assess the retrieval quality, an information content analysis of the different satellite observations, an analysis of the methods and benefits of observation synergy as well as a first assessment of the retrieval uncertainty. Subsequently, section 4 provides a more detailed evaluation of the generated soil moisture estimate, presenting a comparison against modeled soil moisture and in situ soil moisture observations. Finally, section 5 summarizes the results found and gives an outlook on future developments regarding the retrieval product as well as potential studies utilizing the calculated soil moisture database.

\section{Data Sets}

[6] Observations at microwave frequencies have been found to be most sensitive to soil moisture. This is because they are essentially sensitive to the soil dielectric properties, in particular the dielectric constant. Since the dielectric constant of water and bare soil is profoundly different (approximately 80 for water and approximately 3 for dry soil) [Schmugge et al., 1986], the presence of water in the soil greatly affects the combined wet soil dielectric constant, which is clearly visible in microwave observations. However, microwave signals are also influenced by the surface temperature (passive microwave observations), the vegetation cover (passive and active observations), the surface roughness, and other parameters. The purpose of the proposed neural network retrieval scheme is to combine microwave observations with observations at other wavelengths in order to account for the various effects and thus improve the resulting retrieval product. Another reason for using multi-wavelength observations is that the data used in addition to the microwave observations, such as for example the amplitude of the surface temperature, have also been shown to be highly related to soil moisture [Schmugge et al., 1980] and could thus serve as soil moisture proxies as well [Prigent et al., 2005]. This is not only particularly useful in situations where no microwave observations are available, but also greatly helps from an additive synergy point of view. The data sets employed during the retrieval and the processing applied to each of them are discussed in the following paragraphs.

\subsection{Backscatter From the European Remote Sensing (ERS) Satellite}

[7] The ERS scatterometer is an active microwave instrument measuring the backscatter $[\mathrm{dB}]$ at $5.3 \mathrm{GHz}$ and was flown on both ERS satellites. The scatterometer consists of three different antennas, observing at azimuth angles of $45^{\circ}, 90^{\circ}$, and $135^{\circ}$ with respect to the flight path. Each antenna performs a scan across the swath of the flight path [Francis et al., 1991]. Because of this viewing geometry, the local incidence angle of the measurements varies between antennas and between subsequent measurements. In order to obtain a data set in which the individual measurements are comparable to each other, a linear regression has been performed to express the measured backscatter as a function of the incidence angle. This regression function was then used to extrapolate all measurements to a common incidence angle of $40^{\circ}$ in a manner similar to the one proposed by Wagner [1998], reducing the influence of the 
vegetation cover on the signal. For the remainder, the hence transformed ERS data will be denoted by $\sigma_{40}$.

[8] It has been considered to additionally use a soil moisture proxy index that relates to the temporal evolution of the soil moisture similar to the one computed with the change detection algorithm proposed by Wagner [1998]. It is expected that this might improve the retrieval ability to capture the soil moisture temporal variability. However, this algorithm requires a posteriori information on the maximum and minimum soil moisture at a location, which at this point could only have come from a model. To keep the inputs purely model independent, it has thus been decided to use only the backscatter. However, in the future a change detection index might be computed using SMOS data to provide the extreme soil moisture values per location.

\subsection{Surface Emissivities From SSM/I}

[9] Passive microwave sensors are essentially sensitive to changes in the soil dielectric properties, such as the dielectric constant. As explained before, the presence of water in the soil greatly affects the soil dielectric constant, which leads to a high sensitivity of passive microwave sensors to soil moisture. The Special Sensor Microwave/Imager (SSM/I) is a passive microwave radiometer observing at frequencies between 19.35 and $89 \mathrm{GHz}$. Since low-frequency radiation penetrates deeper into the soil, only the $19.35 \mathrm{GHz}$ channels of SSM/I are considered for the soil moisture retrieval. Theoretically frequencies above $10 \mathrm{GHz}$ should show a limited potential for soil moisture retrieval, however, Vinnikov et al. [1999] showed that a soil moisture signal can be retrieved from microwave observations at $18 \mathrm{GHz}$ or above. Observations at the wavelength of $19.35 \mathrm{GHz}$ are performed both at horizontal as well as vertical polarizations, measuring the brightness temperature $T_{B}$ in units of Kelvin [K]. These measurements depend, among other factors, on the state of the atmosphere and the surface temperature. In order to obtain a surface-related parameter, the atmospheric contribution as well as the modulation by the surface temperature was removed and the brightness temperatures were converted to surface emissivities as shown by Prigent et al. [1997]. The two sets of emissivities - at horizontal and vertical polarizations - will be denoted by $e_{h}$ and $e_{v}$, respectively, in this paper.

\subsection{Amplitude of Diurnal Cycle of Surface Temperature}

[10] The temperature product used in the retrieval is the global skin surface temperature data set of the International Satellite Cloud Climatology Project (ISCCP) corrected for atmospheric effects and as processed by Rossow and Schiffer [1999]. The original three-hourly data set was interpolated to obtain the full diurnal cycle as shown by Aires et al. [2004], which was then used to compute the diurnal cycle amplitude. The temperature data have been included in the retrieval, because it has been found that an increase in soil moisture leads to an increase of the thermal inertia of the soil and hence results in a lower diurnal cycle amplitude [Schmugge et al., 1980]. As such the amplitude of the diurnal temperature cycle can be used as a direct proxy for the soil moisture. This is especially advantageous in cases where no microwave data are available. The temperature amplitude measurements are provided in units of Kelvin [K] and will be denoted by $T_{S}$.

\subsection{Normalized Difference Vegetation Index (NDVI)}

[11] In order to account for the effects of vegetation on the microwave observations, a set of global Normalized Difference Vegetation Index (NDVI) data is included in the analysis. In some regions of the Earth, where a strong dependence of vegetation on soil moisture exists, such as savannas, this data set might also serve as a direct proxy for soil moisture. The data are based on infrared and visible spectrum observations of the Advanced Very High Resolution Radiometer (AVHRR) [Gutman, 1999] and will be indicated by NDVI.

\subsection{ORCHIDEE Soil Moisture}

[12] The neural network technique employed for this soil moisture retrieval requires a set of target data in order to be trained. Here the soil moisture of the land surface model Organizing Carbon and Hydrology in Dynamic Ecosystems (ORCHIDEE) developed by the Laboratoire de Météorologie Dynamique (LMD) in Paris [de Rosnay et al., 2002] will be used. The soil moisture is specified in $\left[\mathrm{kg} \mathrm{m}^{-2}\right]$ for 11 layers, the upper four used here representing a total surface depth of $2 \mathrm{~cm}$. This decision has been made since it is assumed that the satellite observations are not sensitive to layers deeper than $2 \mathrm{~cm}$. For the majority of the analysis presented here, the ORCHIDEE soil moisture has been obtained from forcing the model with data from the WATCH project [Weedon et al., 2011]. As part of the evaluation analyses, an additional run of ORCHIDEE forced with ERA-interim data [Balsamo et al., 2010] will be used. The two data sets will be denoted

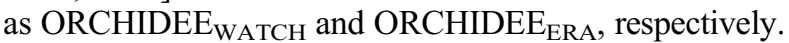

\subsection{HTESSEL Soil Moisture}

[13] For evaluation, the results obtained with the ORCHIDEE runs are also compared against a third independent model soil moisture coming from the Hydrology-Tiled ECMWF Scheme for Surface Exchange over Land (H-TESSEL) model forced with ERA-interim data. It has been decided to use HTESSEL instead of ERA-interim, because it uses updated infiltration relationships and accounts for different soil textures and infiltration capacities [Balsamo et al., 2009]. The volumetric soil moisture $\left[\mathrm{m}^{3} \mathrm{~m}^{-3}\right]$ is specified for four layers, the uppermost used here representing a soil depth of $7 \mathrm{~cm}$. This data set will be denoted by HTESSEL.

\subsection{Pre-Processing}

[14] In order to use the satellite observations as well as the model soil moisture in the proposed retrieval scheme, all data sets have been projected to an equal area grid with a cell center spacing of $0.25^{\circ}$ at the equator and were averaged on a monthly time scale. In addition to that, all pixels covered by snow or ice have been removed from the analysis using the data set of Armstrong and Brodzik [2005]. Pixels containing standing water or closer than $100 \mathrm{~km}$ to a coast were also excluded using the wetland data set of Prigent et al. [2007]. The lower time limit (1993) of the processed 
data is constrained by the flag data availability and the upper limit (1999) is set by the model forcing data.

\section{Retrieval Methodology Development}

[15] The retrieval algorithm is based on Neural Networks $(\mathrm{NN})$. The $\mathrm{NN}$ algorithm represents a nonlinear regression tool used to determine and exploit the general statistical relationship between the satellite observations and soil moisture. The algorithm is used in combination with a set of multi-wavelength satellite observations, each one carrying a different information content on soil moisture variability. It is presumed that the retrieval quality can be greatly improved through the synergy of these observations; however, the improvement is likely to depend on the exact synergy method chosen. The proposed retrieval algorithm performance will depend on the configuration of satellite observations, and thus a tool is proposed to quantify the retrieval uncertainty in different regions of the Earth.

\subsection{Neural Network Algorithm}

[16] The retrieval methodology is based on a neural network algorithm, which uses a combination of microwave (active and passive), infrared, and visible observations as input and computes a corresponding soil moisture.

[17] In the context of remote sensing retrievals neural networks are in essence a non-linear regression tool. In this study, we use the classical feed forward model, as discussed for example in Bishop [1995], consisting of an input layer, an output layer, and a variable number of so-called hidden layers. Each layer contains a number of neurons (or computing nodes) associated with a certain transfer function. A hidden-layer neuron receives its input from one or more neurons of the previous layer, applies the transfer function, and distributes its output to one or more neurons in the subsequent layer. The connections between the neurons are assigned weights, which determine the contribution of one neuron output to another neuron input. The number of neurons in the input and output layer is determined by the number of inputs and outputs, respectively. The number of neurons in the hidden layer(s) depends on the complexity of the problem. It has been shown by Cybenko [1989] that a neural network of sufficient complexity can approximate any continuous function. Because the neural network is able to relate a given output value to any number of input values and because of its ability to learn nonlinear functions, it is very well suited for the fusion of multi-sensor satellite observations in this soil moisture retrieval.

[18] In principle, the neural network determines the global relationship between the inputs and the outputs, i.e., it learns to associate a certain combination of inputs with a certain output. This learning process is known as training and is accomplished by presenting the network with the input values as well as the desired target values. Here the input data are the active and passive microwave observations, the amplitude of the diurnal cycle of the surface temperature and observations of the NDVI. The target data are the modeled soil moisture from ORCHIDEE. It is planned to eventually use SMOS soil moisture as the target data to remove any dependence of the retrieval on the model and to create a product that is consistent with SMOS soil moisture, but covers earlier years. Given the set of inputs, the neural network will compute a set of output soil moisture according to the transfer function and weights specified for the neurons. In the back propagation training scheme [Rumelhart and Chauvin, 1995] used here, these outputs are then compared to the target values, i.e., the ORCHIDEE soil moisture, and a cost function between the two is estimated, which in this analysis is the mean squared error. This mean squared error is a function of the network weights and the purpose of the training step is to adjust the network weights until this cost function has been minimized. In that sense, the node weights can be regarded as nonlinear regression parameters [Rochester et al., 1956]. Once the network has been trained, it can be used with a new set of satellite inputs in order to compute the corresponding soil moisture. Obviously, the ability of the neural network to predict scenarios from a set of input data depends on the versatility of the data set it has been trained with. In other words, a neural network is not able to predict a scenario that is very different from the scenarios in the training data set. For this reason, the training data set should not only be sufficiently large, but also more importantly representative of the expected range of scenarios.

[19] There are some important aspects regarding the operating principle of neural networks. The first one is that during the training step no information on the location or the acquisition time of the data points is provided to the network. As a result, when computing a soil moisture estimate from the trained network, the spatial and temporal structures of this estimate are mainly driven by the observations, not by the model.

[20] Second, it should be kept in mind that any variability present in the input data that cannot be related to variations in the target data will be discarded. Similarly, any variability in the target data that cannot be related to the input data will not be reproduced when using the network to compute estimates. This means that significant differences can exist between the estimates computed from a neural network and the target data that were used to initially train the network. In essence, these differences can have two causes: (1) the soil moisture variability in the target data is unrealistic or (2) the input data are lacking information that is necessary to capture the variability of the target data. Having only the target data and the computed estimates available, it is not possible to determine which of these two factors causes the differences, but a comparison with an independent data set is required.

[21] The range of the retrieved soil moisture will be reduced compared to the original target soil moisture, because the input observations do not provide enough information to characterize extreme events. In order to alleviate this effect the cumulative distribution function (CDF) of the retrieval data has been matched to that of the target data (CDF-matching) [Horn and Woodham, 1979]. In essence, the effect of this transformation is that, e.g., the $5 \%$ of the retrieval points with the lowest values fall within the same soil moisture range as the lowest $5 \%$ of the model soil moisture data. Since the location of the points with the lowest values is different in both data sets, this transformation does not affect the spatial and temporal structures of the retrieved soil moisture, but only the dynamic range of the data. Hence, the results of the correlation analysis will not be affected by CDF-matching. However, since it 
represents a modification of the optimal solution found by the neural network, it will slightly increase the RMS error with respect to the target data.

\subsection{Quality Metrics}

[22] For the subsequent analyses, different quality metrics have been estimated. In the information content and synergy analyses, the metrics are estimated with respect to the training model soil moisture and are used as proxies for the information content carried by the various satellite observations. In the retrieval evaluation, the quality metrics are estimated with respect to modeled soil moisture, other retrieval products, and in situ data, respectively. In these analyses, the metrics serve to estimate the ability of the retrieval to capture different spatial and temporal soil moisture variabilities. In the descriptions below, the data sets with respect to which the metrics are estimated are often referred to as "target data." However, this should not be confused with the target data from the network training step.

RMS error: The root mean squared (RMS) error gives a first indication of the quality of the soil moisture estimate. Since it considers the mean as well as the standard deviation of the estimate errors, the RMS error indicates whether a bias exists between the estimates and the targets and how large the spread of the estimated values around the "true" target values is.

Uncertainty $\sigma$ : The uncertainty $\sigma$ is defined here as the standard deviation of the estimate errors. It provides an indication of the spread of the estimates around the target values and thus signifies how certain the estimates are. It does not provide any information about whether or not a bias exists between the estimates and the targets.

Spatial correlation $\rho_{\text {spatial }}$ : The spatial correlation quantifies the retrieval ability to capture the soil moisture spatial variability of the target data. It is computed by converting the soil moisture for all pixels and the complete time series into a vector and determining the Pearson correlation with respect to the corresponding model data vector. This yields one spatial correlation value per retrieval.

Seasonal correlation $\rho_{\text {seasonal }}$ : The seasonal correlation provides an indication of the retrieval ability to capture the soil moisture seasonal variability. The seasonality of the soil moisture is location dependent and has thus been estimated for each pixel separately. The Pearson correlation between the complete estimate time series of each pixel and the respective target time series was determined. This approach means that the correlation computed is contaminated by the interannual variability, however, this variability is negligible compared to the seasonal variability. The result is a map of seasonal correlations for each of the retrieval scenarios. By their nature, these maps give a rather qualitative measure for the performance of the various retrieval products and it is difficult to compare them directly. In order to obtain a more quantitative measure, a mean seasonal correlation was computed based on these maps. It is obvious that a seasonal correlation only has a physical meaning if there is in fact a sufficiently strong variability to be captured. For this reason, it has been decided to only consider pixels, for which the seasonal cycle amplitude of the soil moisture is larger than $15 \%$ of the maximum amplitude. Additionally, when computing the seasonal correlation maps, the $p$-value of the correlation, specifying the probability that the correlation value was obtained by chance, was estimated in each pixel. For plotting the maps as well as when computing the mean seasonal correlation, pixels with a $p$-value higher than $10 \%$ were removed from the analysis. After this filtering, approximately $44 \%$ of the original data are still included in the analysis.

Interannual correlation $\rho_{\text {interannual }}$ The interannual correlation was determined in order to estimate the ability of the retrieval to capture interannual variations of soil moisture. As for the seasonality, the interannual variation is location dependent and thus the analysis was performed per pixel. To this end, the absolute soil moisture values were converted into anomalies from the mean seasonal cycle in each pixel, and the Pearson correlation with respect to the corresponding target data vector was computed. The mean seasonal cycle in each pixel has been computed by averaging all data maps for a certain month and thus computing a mean map for that month. The result of the correlation computation is a map of interannual correlations for each of the retrieval products. As for the seasonal correlations, a spatial mean correlation value was computed in order to obtain a more quantitative and directly comparable measure of the retrieval capability to capture interannual variations. Again only pixels were considered in which the interannual variability is larger than $15 \%$ of the maximum interannual variation. As for the seasonal correlation, pixels with a $p$-value higher than $10 \%$ have been removed from the maps and any further analysis. After filtering, approximately $49 \%$ of the original points are still considered in the analysis.

\subsection{Information Content of Satellite Observations}

[23] As part of the retrieval algorithm development, an information content analysis of the individual satellite input observations has been performed. To this end, several retrievals were performed using only one of the satellite observations at a time. The quality metrics between the estimates and the ORCHIDEE WATCH $_{\text {soil moisture were }}$ then computed in each case. This served to determine what type of information on soil moisture variability is carried by each of the satellite observations.

[24] Table 1 summarizes the quality metrics obtained for the estimates computed from individual satellite observations. For all quality metrics, the ERS backscatter measurements and the surface temperature diurnal cycle amplitudes provide the best results. In particular, the backscatter data seem to be most suited to capture the seasonal and interannual variability of the soil moisture. These results are not surprising, since microwave observations are known to be the most sensitive to soil moisture. Since the soil properties

Table 1. Quality Metrics Between Estimates Computed From Individual Satellite Observations Using an ORCHIDEE ${ }_{\text {WATCH }}$ Trained NN and ORCHIDEE WATCH $_{\text {Modeled Soil Moisture }}{ }^{\mathrm{a}}$

\begin{tabular}{lccccc}
\hline Data Set & RMSE $\left[\mathrm{kg} \mathrm{m}^{-2}\right]$ & $\sigma\left[\mathrm{kg} \mathrm{m}^{-2}\right]$ & $\rho_{\text {spatial }}$ & $\rho_{\text {seasonal }}$ & $\rho_{\text {interannual }}$ \\
\hline$\sigma_{40}$ & $\mathbf{0 . 9 5 0 7}$ & 1.09 & 0.76 & $\mathbf{0 . 6 5}$ & $\mathbf{0 . 5 6}$ \\
$T_{S}$ & 0.96 & $\mathbf{1 . 0 6}$ & $\mathbf{0 . 7 9}$ & 0.59 & 0.47 \\
$e_{h}, e_{v}$ & 1.00 & 1.14 & 0.74 & 0.46 & 0.39 \\
NDVI & 1.05 & 1.19 & 0.68 & 0.49 & 0.39
\end{tabular}

\footnotetext{
${ }^{\mathrm{a}}$ The best value for each of the quality metrics is shown in bold face.
} 
can react very rapidly to a change of soil moisture, the ERS backscatter is best suited to capture the soil moisture temporal variability. It is expected that if an index based on change detection as proposed by Wagner [1998] would be used in addition to the raw backscatter, this ability to capture temporal soil moisture variability could even be further improved.

[25] Table 1 also shows a good performance of the temperature-based retrieval product, especially with regard to the spatial variability of the soil moisture. This is because the surface temperature can be very heterogeneous and is thus well suited to pick up soil moisture spatial variability. These results underline that the amplitude of the diurnal temperature cycle serves as a good proxy for the soil moisture itself. As discussed before, this is due to the influence of the soil moisture on the soil thermal inertia and evaporation and hence on the amplitude of the diurnal cycle.

[26] Based on intuition it could be expected that NDVI would perform best in capturing the soil moisture seasonal variability due to the obvious relationship between water availability and plant growth. While the seasonal correlation obtained from an NDVI-only retrieval is high, the temperature and active backscatter product perform better. It is possible that the correlation value obtained with the NDVI product could be increased if temporal lags were considered in the analysis, but the lags would most likely differ from one location to another. Another possibility is that the NDVI product might be slightly contaminated by clouds in some regions, leading to a seasonal correlation that is lower than expected. However, it should also be noted that in the midlatitudes, the NDVI is more strongly driven by the temperature and the length of the day and hence no strong correlation with the soil moisture should be expected in these regions.

\subsection{Synergy Analysis}

[27] One objective of this study is to assess to what extent a retrieval benefits from the use of multiple observations and which synergy method yields the largest improvement.

\subsubsection{Stepwise Observation Combination}

[28] The first point has been analyzed by combining the observations in a stepwise approach. This means that the individual observation with the best quality metrics was selected and subsequently combined with each of the remaining observations, respectively. A new set of soil moisture estimates has been computed from these combinations of two and the combination with the best quality metrics was chosen. In this manner, a new observation was added to the best combination upon each iteration [Efroymson, 1960]. This iterative approach is preferred over a simple combination of the best individual observations in order to ensure that the most complementary combination is chosen.

[29] Table 2 shows the quality metrics for the best individual data set and the best combinations. In terms of RMS error and uncertainty the quality of the retrieval increases with each data set added and - as expected - the combination of all data sets yields the optimal result. This means that for a larger combination of data sets the information content is higher and hence the retrieval will be of a higher quality. The results also clearly illustrate the effects of complementarity discussed before. While the ERS backscatter and
Table 2. Quality Metrics Between Estimates Computed From Observation Combinations of Different Sizes Using an ORCHIDEE $_{\text {WATCH }}$ Trained NN and ORCHIDEE WATCH $_{\text {Modeled Soil }}$ Moisture

\begin{tabular}{lccccc}
\hline Data Sets & RMSE $\left[\mathrm{kg} \mathrm{m}^{-2}\right]$ & $\sigma\left[\mathrm{kg} \mathrm{m}^{-2}\right]$ & $\rho_{\text {spatial }}$ & $\rho_{\text {seasonal }}$ & $\rho_{\text {interannual }}$ \\
\hline$\sigma_{40}$ & 0.95 & 1.09 & 0.76 & 0.65 & 0.56 \\
$\sigma_{40}$ & 0.89 & 0.98 & 0.81 & 0.61 & 0.46 \\
NDVI & & & & & \\
$\sigma_{40}$ & & & & & \\
NDVI & 0.86 & 0.93 & 0.83 & 0.61 & 0.46 \\
$T_{S}$ & & & & & \\
$\sigma_{40}$ & & & & & \\
NDVI & 0.85 & 0.92 & 0.84 & 0.63 & 0.48 \\
$T_{S}$ & & & & & \\
$e_{h}, e_{v}$ & & & & & \\
\hline
\end{tabular}

the diurnal temperature cycle amplitudes perform best individually, their combination does not yield the best retrieval from a combination of two. Instead, the combination of ERS backscatter and NDVI data yields the best result, which indicates that these two data sets complement each other more.

[30] Regarding the temporal correlations, the retrieval from active microwave data alone actually performs better than the retrievals from combinations. This is because during training the network attempts to minimize the errors between the estimates and the target data and finds the best compromise between the information provided by the different observations. In the case of the soil moisture retrieval, this leads to a degradation of the temporal behavior.

[31] While the seasonal correlations for the combination retrievals are consistently high, adding new observations only slightly increases the retrieval quality. It was also observed that when using points in which the seasonal variability is high, the seasonal correlations increase significantly. This demonstrates that the ability of the retrieval to capture seasonal variability is proportional to the magnitude of the variation.

[32] Only very small improvements in the interannual correlation can be achieved through the addition of observations, which leads to the conclusion that no new information on the interannual variability is added by the data sets. Upon comparison of maps of the interannual variability to maps of the estimate error standard deviation, it was found that in most regions the error variability was higher. This makes it impossible for the retrieval to capture the interannual variability. In regions where the interannual variability was higher, mean correlations of 0.65 could be obtained.

[33] The spatial correlation values are consistently high and increase steadily with the addition of observations. The consistent increase of the correlations in Table 2 indicates that each data set is able to provide some new information about the spatial behavior and thus contributes to an improvement of the retrieval.

\subsubsection{Synergy Factors}

[34] In order to better quantify the retrieval improvement upon each addition of an observation the so-called synergy factor was determined for each of the retrieval products. The synergy factor $S F$ is a measure of the percentage by which a retrieval product is improved through data synergy compared to the best single observation retrieval. It is defined as 


$$
S F=\left(1-\frac{\sigma_{\text {combination }}}{\sigma_{\text {best,individual }}}\right) * 100,
$$

where $\sigma_{\text {combination }}$ is the uncertainty of the combination based retrieval and $\sigma_{\text {best,individual is the uncertainty of the }}$ best individual retrieval.

[35] In general, there are two options to produce a multiobservation retrieval product as discussed by Aires et al. (submitted manuscript, 2011): (1) data fusion, where the retrieval is performed on the combined set of observations, as in the methodology proposed here, and (2) a posteriori combination, which designates a weighted averaging of retrieval products from individual observations. As part of the synergy analysis, it has been investigated to what extent the data fusion approach compares to the a posteriori combination. To this end the a posteriori combinations have been computed using

$$
f_{1: n}=\frac{\sum_{i=1}^{n}\left(\frac{\prod_{k=1}^{n} \sigma_{k}^{2}}{\sigma_{i}^{2}} f_{i}\right)}{\sum_{i=1}^{n}\left(\frac{\prod_{k=1}^{n} \sigma_{k}^{2}}{\sigma_{i}^{2}}\right)}
$$

where $n$ is the number of retrieval products to be combined, $f$ denotes the retrieval, and $\sigma$ is the uncertainty associated with each retrieval product. The theoretical uncertainty of the resulting estimates is given by

$$
\sigma_{1: n}=\frac{\prod_{i=1}^{n} \sigma_{i}}{\sqrt{\sum_{i=1}^{n}\left(\frac{\prod_{k=1}^{n} \sigma_{k}^{2}}{\sigma_{i}^{2}}\right)}} .
$$

[36] This theoretical uncertainty as well as the true uncertainty of the a posteriori combinations were determined and compared to the uncertainty of the data fusion estimates. In addition, the synergy factor of the a posteriori combinations with respect to the best individual retrieval was computed and compared to the synergy factors obtained from the data fusion estimates.

[37] Table 3 shows the uncertainty of the data fusion based retrieval $\sigma_{\mathrm{DF}}$, the uncertainty of the best individual retrieval

Table 3. Uncertainties (in $\mathrm{kg} \mathrm{m}^{-2}$ ) and Synergy Factors for Estimates Computed From the Best Combinations of Different Size ${ }^{\mathrm{a}}$

\begin{tabular}{lcccccc}
\hline Data Sets & $\sigma_{\text {combination }}$ & $\sigma_{\text {best,individual }}$ & $\sigma_{\mathrm{AP} \text {,theory }}$ & $\sigma_{\mathrm{AP}, \text { true }}$ & $S F_{\mathrm{DF}}$ & $S F_{\mathrm{AP}}$ \\
\hline$\sigma_{40}$ & 0.97 & 1.09 & 0.80 & 1.00 & 10.59 & 8.50 \\
NDVI & & & & & & \\
$\sigma_{40}$ & & & & & & \\
NDVI & 0.93 & 1.06 & 0.64 & 0.98 & 12.88 & 8.06 \\
$T_{S}$ & & & & & & \\
$\sigma_{40}$ & & & & & & \\
NDVI & & & & & \\
$T_{S}$ & 0.92 & 1.06 & 0.55 & 1.05 & 13.41 & 1.44 \\
$e_{h}, e_{v}$ & & & & & &
\end{tabular}

\footnotetext{
${ }^{\mathrm{a}}$ The estimates are computed using an ORCHIDEE ${ }_{\text {WATCH}}$-trained net-

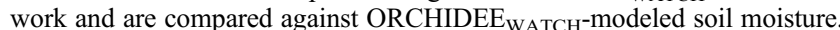
Uncertainty of the combination based retrieval $\boldsymbol{\sigma}_{\mathrm{DF}}$, the uncertainty of the best individual retrieval $\boldsymbol{\sigma}_{\text {best,individual, }}$ the theoretical uncertainty of the a

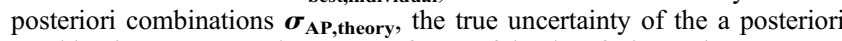
combinations $\boldsymbol{\sigma}_{\mathrm{AP} \text {,true }}$, the synergy factor of the data fusion estimates $S F_{\mathrm{DF}}$ and the synergy factor of the a posteriori combination estimates $S F_{\mathrm{DF}}$.
}

$\sigma_{\text {best,individual, }}$ the theoretical uncertainty of the a posteriori

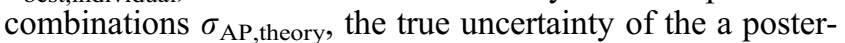
iori combinations $\sigma_{\mathrm{AP} \text {,true }}$ (which is defined as the standard deviation of the estimate errors), the synergy factor of the data fusion estimates $S F_{\mathrm{DF}}$, and the synergy factor of the a posteriori combination estimates $S F_{\mathrm{AP}}$.

[38] When comparing the retrieval based on the ERS backscatter data with that based on the combination of all data sets, a decrease in the uncertainty of over $13 \%$ can be observed, which supports the decision to perform multiwavelength observation retrieval.

[39] For each combination, the data fusion synergy factors are higher, indicating that a data fusion approach improves the retrieval product more significantly than an a posteriori combination. This result is very important with regard to the objective of combining the best aspects of different retrieval methodologies. It indicates that the strategy should be to merge the data sets within a single algorithm rather than simply combining the existing retrieval products.

[40] Another important result to be observed is that in every case the theoretical uncertainty is significantly lower than the true uncertainty. This is because the theoretical estimation assumes that the individual uncertainties are independent and follow a Gaussian distribution, which might not be the case.

\subsection{Retrieval Uncertainties}

[41] As a tool to estimate the retrieval uncertainty in different regions at different times, monthly maps of the signal-to-noise ratio (SNR) have been computed. These represent a simple and innovative way to estimate the retrieval uncertainties. To achieve this, distinct satellite input configurations were separated into bins. To define the bins, the space between the minimum and maximum value of each input variable was divided into 10 intervals. These were then combined into a five-dimensional array (one dimension for each input variable) of bins with each bin corresponding to one combination of input variables. Next, the correct bin was determined for each data point and the model soil moisture value corresponding to that data point was added as an entry to the bin. As a final step, the standard deviation of the soil moisture values collected in each bin was determined, yielding one standard deviation value per bin. This standard deviation represents the variation of modeled soil moisture for the same combination of satellite inputs and is thus a measure of the uncertainty of the retrieval. In the next step, for the satellite observations for each year, each month, and each pixel, the correct bin was determined and the corresponding soil moisture standard deviation was assigned to the pixel, yielding one map of soil moisture standard deviations per month per year. These were then converted into maps of signal-to-noise ratio by dividing the seasonal cycle amplitude of each pixel (the signal) by the soil moisture variability for the given input combination (the noise).

[42] Figure 1 shows monthly mean maps of the signal-tonoise ratio. It can be seen that regions of low SNR are the arid regions, parts of the Amazon tropical forest as well as parts of the northern U.S. and some regions in China and Siberia. In further analyses of the retrieval (presented in section 4), it was found that in the arid and tropical forest regions the retrieval is still able to perform well. This is 

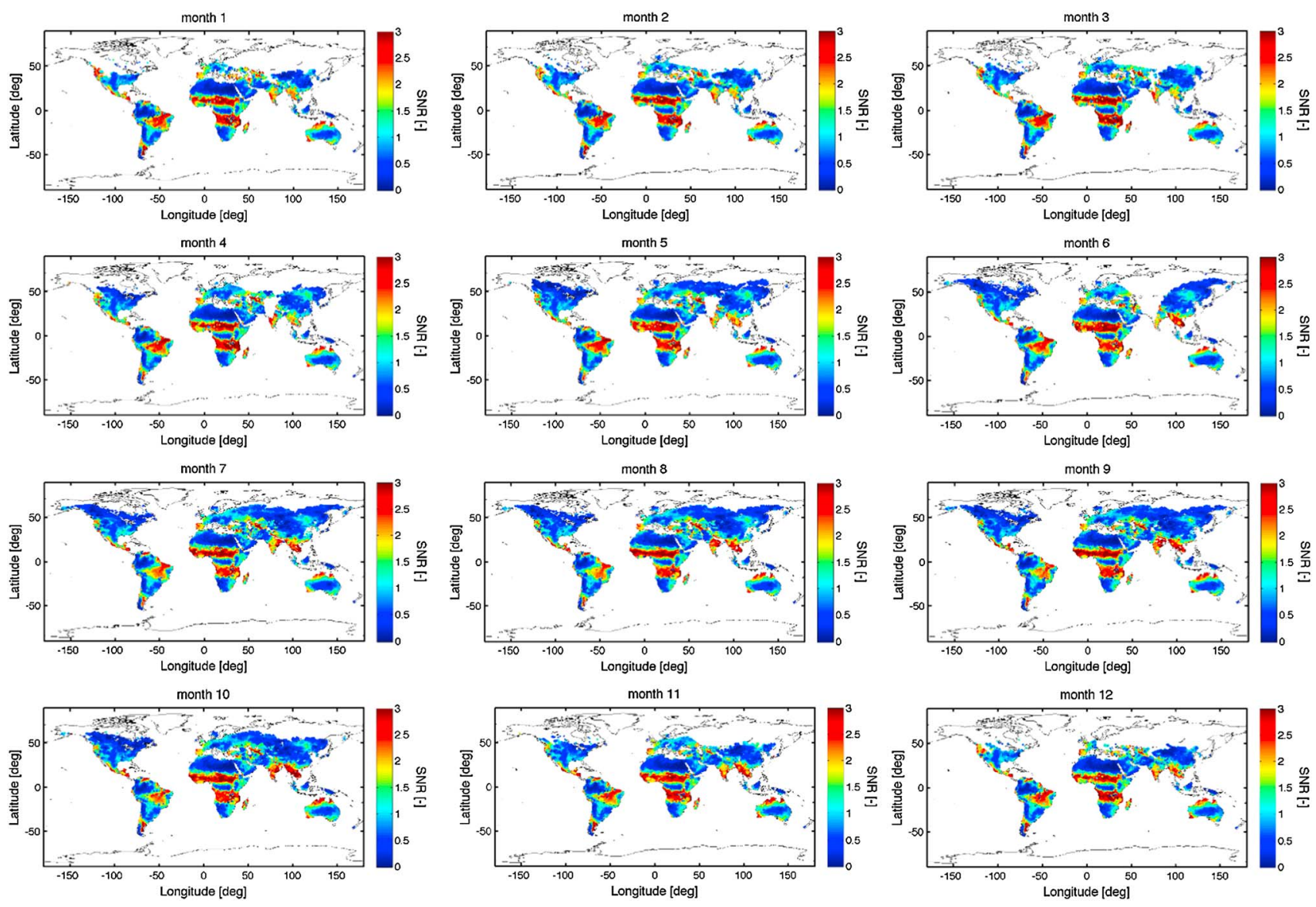

Figure 1. Monthly maps of mean signal-to-noise ratio (SNR). The signal-to-noise ratio is defined as the soil moisture seasonal cycle amplitude for a certain pixel divided by the soil moisture standard deviation for the observed satellite data configuration.

likely due to the fact that the soil moisture signal is more or less flat in these regions and can thus easily be estimated. However, in regions with a low SNR and a discernible seasonal cycle (the northern U.S. and parts of Asia), the proposed retrieval methodology will not be able to estimate the correct soil moisture. These results are particularly interesting with regard to other soil moisture retrieval products, which often show large uncertainties over densely vegetated or arid regions, while in the US and Asia they tend to perform better than the retrieval proposed here. This means that the proposed methodology has a tendency to complement existing retrievals.

\section{Evaluation}

[43] The ability of the estimated soil moisture to capture different soil moisture variabilities has been evaluated by comparing it to modeled and in situ soil moisture.

\subsection{Modeled Soil Moisture}

[44] In the first phase of the retrieval product evaluation, the estimated soil moisture has been compared against different modeled soil moisture fields. Those are the HTESSEL soil moisture as well as two ORCHIDEE runs, ORCHIDEE WATCH $_{4}$ and ORCHIDEE $E_{\mathrm{ERA}}$. For each model and the corresponding retrieval product, the spatial, seasonal, and interannual correlations have been estimated and are shown in Table 4 . A large spread of the correlation values for the different models exists, which indicates a varying level of consistency between the retrievals and their respective training models. The spatial and temporal structures of the estimated soil moisture are driven by the observations, so the level of inconsistency of a retrieved soil moisture with its training model can be translated directly into inconsistencies between the model and the observations.

[45] In order to verify this assumption, the correlations between two of the retrieval products as well as between the two corresponding training models have been estimated. The results of this analysis are shown in Table 5. Clearly, the

Table 4. Spatial, Seasonal, and Interannual Correlations Between the Retrieval Products and Their Respective Training Model ${ }^{\mathrm{a}}$

\begin{tabular}{lccc}
\hline Quality Metric & ORCHIDEE $_{\text {WATCH }}$ & HTESSEL & ORCHIDEE $_{\text {ERA }}$ \\
\hline$\rho_{\text {spatial }}$ & 0.84 & 0.90 & 0.81 \\
$\rho_{\text {seasonal }}$ & 0.63 & 0.67 & 0.54 \\
$\rho_{\text {interannual }}$ & 0.48 & 0.49 & 0.34 \\
\hline
\end{tabular}

${ }^{\mathrm{a}}$ The first column shows the correlations between the ORCHIDEE $\mathrm{WATCH}_{\mathrm{H}}$ trained retrieval and the ORCHIDEE $\mathrm{WATCH}_{\mathrm{W}}$ model soil moisture. The second column displays the correlations between the HTESSEL trained retrieval and HTESSEL. The third column displays the quality metrics between ORCHIDEE $\mathrm{ERA}_{\mathrm{EA}}$ and the retrieval trained with it. 
Table 5. Spatial, Seasonal, and Interannual Correlations ${ }^{\mathrm{a}}$

\begin{tabular}{lcc}
\hline Quality Metric & Retrieval Products & Model Products \\
\hline$\rho_{\text {spatial }}$ & 0.91 & 0.80 \\
$\rho_{\text {seasonal }}$ & 0.88 & 0.74 \\
$\rho_{\text {interannual }}$ & 0.76 & 0.70 \\
\hline
\end{tabular}

${ }^{a}$ Between the retrieval products trained with ORCHIDEE $_{\text {WATCH }}$ and HTESSEL (left column) and between the original ORCHIDEE WATCH $_{\text {and }}$ HTESSEL model soil moisture products (right column)

two retrieval products are much more similar than the two models. This supports the assumption that the spatial and temporal structures of the estimated soil moisture stem from the observations. Hence, even if dissimilarities between two training models exist, when using the same satellite observations, the two estimated soil moisture fields will be very similar.

[46] From Table 4 it could be seen that large inconsistencies exist between ORCHIDEE $E_{\text {ERA }}$ and the observation driven soil moisture estimate, whereas HTESSEL was found to be very close to the estimate and hence the observations. In a next step of the evaluation process, the estimate from

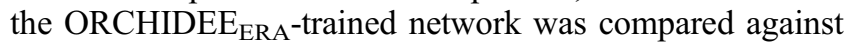
HTESSEL. The results, shown in Table 6, indicate that the estimated soil moisture is much closer to HTESSEL than

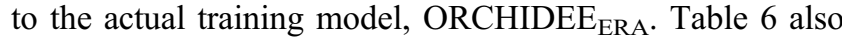
shows the correlations between the two models, which are

Table 6. Spatial and Temporal Correlations ${ }^{\mathrm{a}}$

\begin{tabular}{lccc}
\hline $\begin{array}{l}\text { Quality } \\
\text { Metric }\end{array}$ & $\begin{array}{c}\text { ORCHIDEE } \\
\text { Model; HTESSEL } \\
\text { Model }\end{array}$ & $\begin{array}{c}\text { ORCHIDEE }_{\text {ERA }} \\
\text { Retrieval; } \\
\text { ORCHIDEE }\end{array}$ & $\begin{array}{c}\text { ORCHIDEE }_{\text {ERA }} \text { Model } \\
\text { Retrieval; HTESSEL } \\
\text { Model }\end{array}$ \\
\hline$\rho_{\text {spatial }}$ & 0.79 & 0.81 & 0.82 \\
$\rho_{\text {seasonal }}$ & 0.57 & 0.53 & 0.63 \\
$\rho_{\text {interannual }}$ & 0.31 & 0.34 & 0.45 \\
\hline
\end{tabular}

${ }^{a}$ Column 1: Spatial and temporal correlations between the model

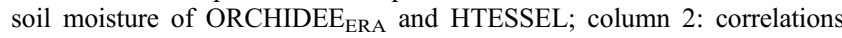

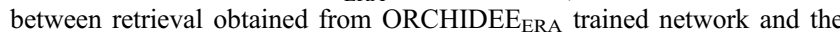
ORCHIDEE $_{\text {ERA }}$ model soil moisture; column 3: correlations between retrieval obtained from ORCHIDEE ERA $_{\text {trained network and the HTESSEL }}$ model soil moisture. much lower, so the closeness of the retrieved soil moisture to HTESSEL cannot be attributed to a closeness of the models. This means that the variability of the retrieval soil moisture is significantly different from the variability of the training model. However, the same variability as in the retrieval product can be observed in the HTESSEL data, which are known to be consistent with the observations. Hence, the variability of the retrieval product clearly originates from the observations. These results are consistent with the findings of Jimenez et al. [2009], who use a similar neural network strategy to create an observation driven land heat flux product.

[47] It should be noted that, generally, discrepancies observed between the estimated soil moisture and the training model can be due to either (1) an error of soil moisture in the model or (2) a lack of information and/or errors in the satellite observations. If the latter was the case, the variability in the estimated soil moisture should be random and not be observed in other soil moisture products. Thus, the fact that the same variability is observed in the HTESSEL soil moisture indicates that the differences observed with respect to ORCHIDEE ERA $_{\text {are most likely }}$ due to soil moisture variabilities in the model that have no equivalent in actual retrieved soil moisture. This shows that the proposed methodology uses the observations in order to correct inconsistencies in the model and thus moves it toward a product that displays higher agreement with observed soil moisture. Since the analysis showed that the HTESSEL soil moisture is most consistent with the observations and since HTESSEL is available for a long time period, it has been decided to use it as the training model for the retrieval in the future.

[48] In order to assess the consistency between the modeled and retrieved soil moisture in different regions of the Earth, maps of the seasonal and interannual correlations as well as of the RMS error are shown in Figures 2-4.

[49] Figure 2 shows the interannual correlation between the retrieved and the ORCHIDEE $\mathrm{WATCH}_{\mathrm{W}}$ soil moisture. While in some regions very high correlations with values close to 1 are obtained, other regions show a zero correlation. Upon comparison of the correlation map to a map of the interannual variability of the ORCHIDEE WATCH $_{\text {soil }}$

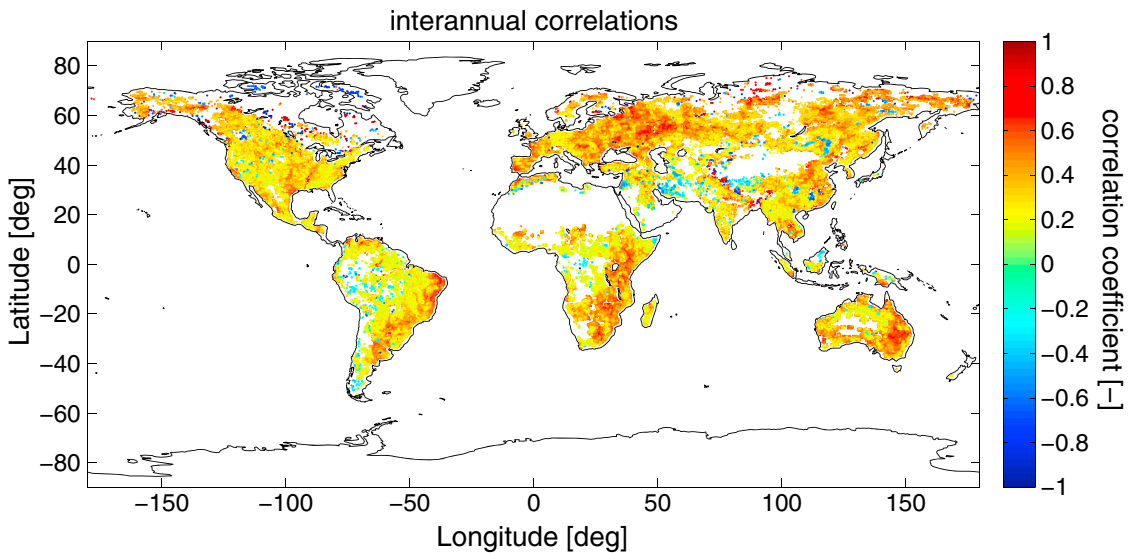

Figure 2. Interannual correlations between soil moisture estimates and ORCHIDEE $\mathrm{WATCH}_{\text {model soil }}$ moisture. White areas represent locations with a correlation that is statistically not significant ( $p$-value higher than 0.1 ) or where the interannual variability is smaller than $15 \%$ of the maximum variability. 


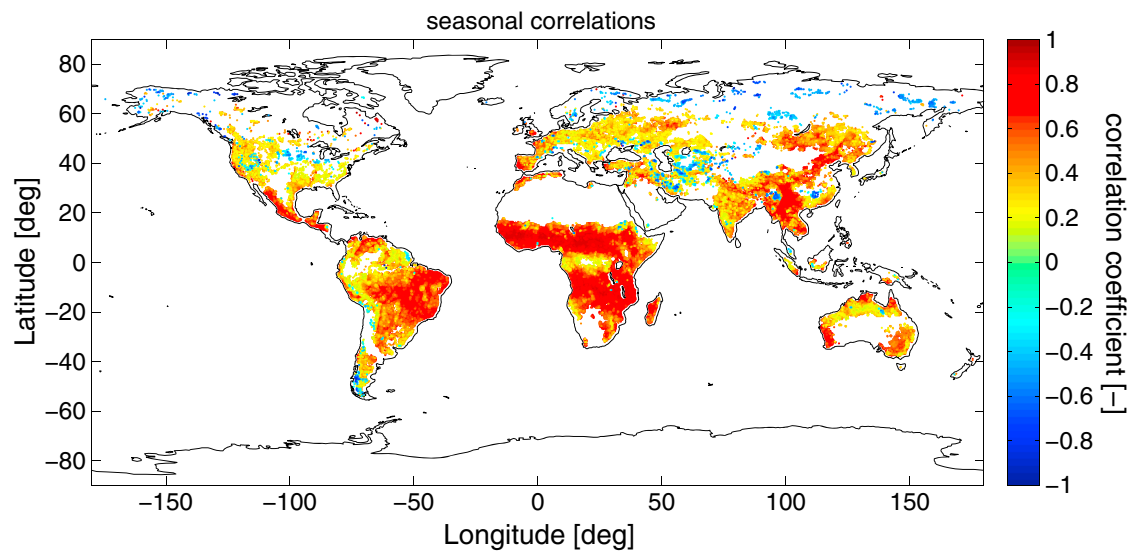

Figure 3. Seasonal correlations between soil moisture estimates and ORCHIDEE WATCH $_{\text {model soil }}$ moisture. White areas represent locations with a correlation that is statistically not significant ( $p$-value higher than 0.1 ) or where the seasonal variability is smaller than $15 \%$ of the maximum variability.

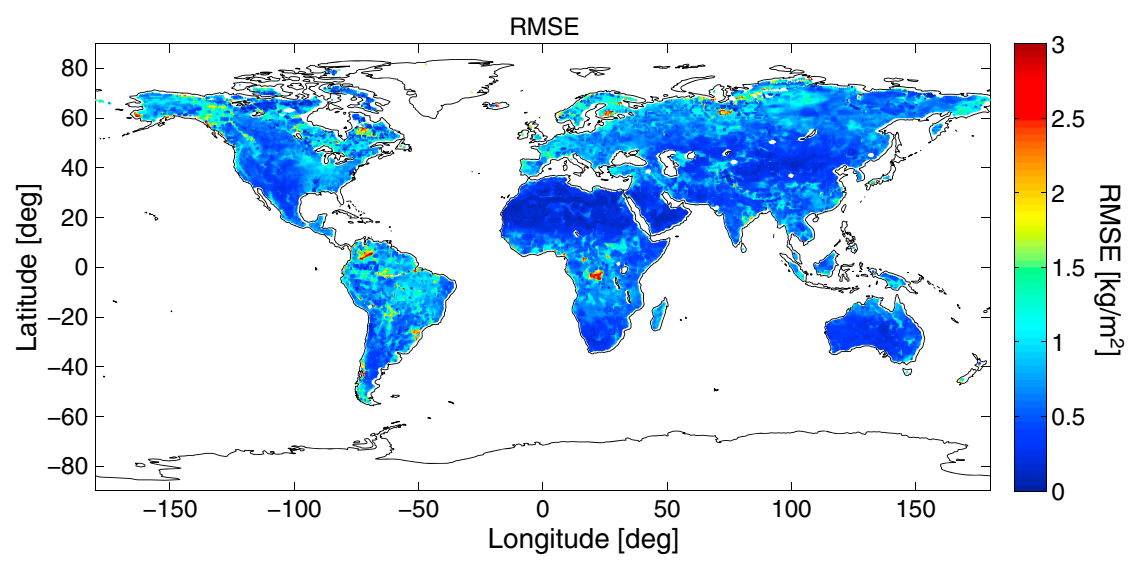

Figure 4. The RMS error between best estimate and ORCHIDEE ${ }_{\mathrm{WATCH}}$ model soil moisture.

moisture (not shown here), it was found that the regions of high interannual correlation correspond to the regions where the interannual variability of the modeled soil moisture is strong. This means that if there is a strong interannual variability, the satellite observations and the retrieval are able to capture it well.

[50] Figure 3 shows the seasonal correlations between the retrieved and modeled soil moisture, which are found to be highly variable. When comparing Figure 3 to a map of the seasonal cycle amplitude, it was observed that in regions where the soil moisture seasonal cycle is strong, a high seasonal correlation is always obtained. This means that in regions with a strong seasonal cycle the retrieval algorithm is able to capture and reproduce it.

[51] For both temporal correlations, it is expected that adding an index related to the temporal evolution of the backscatter as input might enhance the retrieval ability to capture the soil moisture temporal variations and thus improve its temporal performance.

[52] A map of the RMS errors between the retrieved and the modeled soil moisture is shown in Figure 4. While for most of the Earth the RMS error is below $1 \mathrm{~kg} \mathrm{~m}^{-2}$, regions close to large rivers or in areas with many lakes (Congo Basin, Orinoco Delta, Ob River, Canadian lakes, Finnish lakes) show higher errors. The two possible explanations for this are (1) a too lenient filtering of standing water bodies and (2) the fact that pixels, while not inundated might still be saturated, which would lead to very strong satellite signals and a high soil moisture retrieved, which might not be captured in the monthly mean model soil moisture.

[53] It is also evident that the retrieval performs well in arid and densely vegetated regions, which often pose a challenge in soil moisture retrievals. This underlines the potential of the proposed methodology to complement existing retrieval algorithms and it is thus suggested to aim for the incorporation of the beneficial aspects in one algorithm.

\subsection{In Situ Observations}

[54] As a further evaluation step, the estimated soil moisture has been compared to in situ soil moisture observations. The in situ data were retrieved from the International Soil Moisture Network [Dorigo et al., 2011] and originate from different measurement networks, which for the period 1993-1999 cover mainly the U.S., China, and Mongolia. An overview of the network characteristics is given in Table 7. To compare the neural network-based retrieval skills to those of other retrieval products or models, the same analysis has been performed for the HTESSEL soil moisture and the Water Cycle Multimission Observation Strategy (WACMOS) retrieval product [Liu et al., 2011]. 
Table 7. Characteristics of the In Situ Networks Utilized in the Evaluation Analyses ${ }^{\mathrm{a}}$

\begin{tabular}{llcc}
\hline Network Name & \multicolumn{1}{c}{ Location } & No of Stations & Top Layer Depth [m] \\
\hline ARM & Kansas, Oklahoma, Colorado & 25 & 0.025 \\
CHINA & China & 40 & 0.025 \\
ICN & Illinois & 19 & 0.05 \\
IOWA & Iowa & 6 & 0.035 \\
MONGOLIA & Mongolia & 44 & 0.05 \\
SCAN & USA & 182 & 0.025 \\
SNOTEL & Western USA (including Alaska) & 381 & 0.05 \\
\hline
\end{tabular}

${ }^{a}$ Network name, regions of the Earth covered, number of stations in the network, and average depth of the top layer.

[55] The comparison of in situ observations with retrieved or modeled soil moisture is not trivial. This is because the in situ data represents a point measurement of a highly heterogeneous variable, whereas retrieved and modeled soil moisture correspond to averages over large areas of several square kilometers. Additionally, the in situ measurements are often taken at depths different to what model or retrieval data correspond to and even between the various measurement networks, there can be large discrepancies due to the diverse measurement techniques used. All these factors introduce uncertainties in a comparison with in situ data and this should be kept in mind.

[56] For the evaluation performed here, the spatial and the temporal correlation between the estimated soil moisture and the in situ observations have been computed. To this end all in situ data have been averaged to monthly mean values, ignoring pixels where only one measurement was available per month. If more than one in situ station fell into one of the retrieval grid cells, their contributions were averaged. Pixels with a signal-to-noise ratio lower than 1 (cf. section 3.5) have been removed from the comparison.

[57] The spatial correlation was computed for each year and each month in order to remove all temporal variation of the data. Because the various in situ networks measure at different depths and with different techniques, a spatial correlation has been computed for each network separately. Subsequently, the time series of correlations obtained with this method have been averaged to yield one spatial correlation value per network. In this averaging step, pixels for which less than two correlation values were available over the entire time period have been removed. The mean spatial correlations for the two retrieved and the modeled soil moistures are shown in the first three columns of Table 8 .

[58] It can be seen that values are only available for ARM, MONGOLIA, and SCAN. For the other in situ networks either the number of data points was too low (in the case of CHINA and SNOTEL) or they were not considered because of a too low signal-to-noise ratio (the case for ICN and IOWA). For the remaining networks, both the retrieved and modeled soil moisture show a good spatial correlation with the in situ data. Both retrievals are capable to better capture the soil moisture spatial variability over the ARM region and the neural network algorithm also outperforms the model over Mongolia. This indicates that there is a potential for the retrieval products to deliver better knowledge on the soil moisture spatial variability.

[59] The temporal correlation has been computed per pixel using the entire time series available. If less than five data points were available in the time series of a pixel, no correlation has been computed for that pixel. The obtained correlations were then averaged per network to yield one temporal correlation value for each of the networks. In this averaging step, correlations with a $p$-value higher than 0.3 have been removed. The resulting mean correlations are shown in the last three columns of Table 8. The temporal correlations of the modeled soil moisture with the in situ data are always higher than those between the retrievals and the in situ data. This is likely due to the fact that the model utilizes precipitation and other atmospheric data, which greatly help to constrain the temporal behavior. The retrievals on the other hand are limited to the use of surface parameters alone. In China and Mongolia, the correlations for the neural network retrieval are reasonable, albeit lower than those for the model. However, for the stations in the U.S., the retrieval is not able to capture the correct temporal behavior. This agrees with the results from the uncertainty analysis (cf. section 3.5), in which the U.S. has been identified as a region in which the retrieval is uncertain. This is likely the result of a positive correlation between the temperature amplitude and the soil moisture in this region, which contradicts the general relationship the network determined between these two variables and thus causes a flawed estimation. The reason for this positive correlation lies probably in the increased vegetation cover (mainly agricultural crops) during the summer when the soil moisture is at its minimum. Increased vegetation tends to absorb most of the incoming solar radiation for evapotranspiration and

Table 8. Spatial and Temporal Correlations Between In Situ Observations and Estimated and Modeled Soil Moisture ${ }^{\mathrm{a}}$

\begin{tabular}{|c|c|c|c|c|c|c|}
\hline \multirow[t]{2}{*}{ Network } & \multicolumn{3}{|c|}{ Spatial } & \multicolumn{3}{|c|}{ Temporal } \\
\hline & NN Estimate & WACMOS & HTESSEL & NN Estimate & WACMOS & HTESSEL \\
\hline ARM & 0.65 & 0.59 & 0.48 & -0.01 & 0.49 & 0.71 \\
\hline CHINA & - & - & - & 0.34 & 0.44 & 0.67 \\
\hline MONGOLIA & 0.62 & 0.14 & 0.50 & 0.47 & 0.55 & 0.58 \\
\hline SCAN & 0.51 & 0.56 & 0.67 & -0.07 & 0.64 & 0.72 \\
\hline
\end{tabular}

${ }^{a}$ Correlations are shown for soil moisture product estimated with the neural network algorithm, the WACMOS soil moisture retrieval product, and the HTESSEL modeled soil moisture. 
it is thus no longer available for surface heating, leading to reduced temperature cycle amplitude. Generally, the WACMOS retrieval shows a better ability to capture the soil moisture temporal variability. This is because it is largely based on the change detection algorithm proposed by Wagner [1998], which lays a strong focus on the data temporal evolution and calibrates the algorithm for each pixel individually.

[60] The above results support the argument that better retrieved soil moisture could be obtained by combining different aspects of the existing methodologies. The proposed strategy is to add an index related to the time evolution of the backscatter data as input to the neural network. It is expected that this would improve the retrieval's temporal performance, while retaining the good spatial performance shown by the neural network-based algorithm.

\section{Conclusion and Perspectives}

[61] In this paper a soil moisture retrieval methodology has been proposed, which utilizes neural networks in order to combine a set of multi-wavelength satellite observations and which is trained on the HTESSEL modeled soil moisture. The methodology has been proposed as a first step to combine the existing knowledge on soil moisture retrievals and thus improve the retrieval quality. The algorithm proposed in this paper focuses on the combination of all observations with a known sensitivity to soil moisture in an attempt to capture different soil moisture variabilities. It has been shown that this methodology works well in regions, which pose problems for other retrievals and thus the methodology developed here is complementary to existing algorithms.

[62] An information content analysis of the different satellite observations showed that the active microwave data provides the most information about the soil moisture temporal variability, whereas the amplitude of the surface temperature diurnal cycle is best suited to capture the soil moisture spatial variability.

[63] Next, a synergy analysis has been performed, which showed that the combination of all available observations yielded a $13 \%$ decrease of retrieval uncertainty with respect to the best individual retrieval. This underlines that soil moisture retrieval can greatly benefit from the combination of multi-wavelength observations. It was also found that for all combinations, data fusion yielded a much more significant retrieval improvement than a posteriori combination of independent retrieval products. This means that with regard to combining the best aspects of existing retrievals, a simple combination of the existing products is not considered the optimal approach. Instead future work should be directed at developing one single algorithm that combines aspects of the different retrieval strategies.

[64] Finally, the performance of the proposed methodology has been evaluated by comparing the estimated soil moisture with various modeled soil moisture fields and it could clearly be shown that the temporal and spatial structures of the retrieval product are determined by the satellite observations. Furthermore, it was shown that the proposed neural network algorithm is able to detect and correct model soil moisture inconsistencies with the satellite observations.
[65] In the next phase, the estimated soil moisture as well as the HTESSEL soil moisture and the WACMOS retrieval product was compared against in situ observations. It was found that both retrieved soil moisture data sets are well able to capture the spatial variability observed in the in situ observations and even showed the potential to be used for model improvement in most regions. Of the three data sets analyzed, the neural network-based estimate was found to best approximate the observed soil moisture spatial variability. Regarding the temporal variability, the model always obtained the highest correlations; however, this was partly attributed to the fact that the model's temporal behavior is strongly constrained through the atmospheric forcing data. It was found that the WACMOS data set better captures the soil moisture temporal variability. This was attributed to the use of a change detection method, which focuses on the temporal evolution of the observations. It has been concluded that in a new version of the neural network retrieval, information related to the temporal evolution of the backscatter data should be added as one of the input data sets. This would represent the next step in the direction of creating an algorithm that employs the most beneficial aspects of the existing retrieval schemes.

[66] Another step toward this objective would be to calibrate the neural network algorithm on either SMOS or SMAP retrieval data, as soon as the retrieval products have been calibrated and the algorithms fully validated. This would allow the retrieval product to be more independent from models and thus increase its value as a model evaluation data set. Furthermore, it would create a product that is compatible with SMOS data, but covers a different and longer time period.

[67] Up to this point a soil moisture database trained with HTESSEL has been developed that spans the years 1993-2000. The next phase is to extend this data set to a longer time period and thus make it more useful for model evaluation and climatological studies. Based on a survey of the existing satellite observations, the period 1983-2010 is deemed feasible. As part of this data set extension, several analyses will be performed regarding the issues associated with it. In particular, it will be estimated to what extent a missing data set impacts the retrieval quality and it will be investigated whether additional calibration will be necessary when one of the input data sets changes (e.g., from ERS to ASCAT).

[68] Furthermore, the results presented here have been computed from monthly mean soil moistures, which are very useful for long-term analyses and climate studies. The main reason to use monthly means up to this point has been a constraint imposed by the temporal resolution of some of the input data. However, the methodology presented here is equally applicable to data at other timescales and could thus be used to create a soil moisture data set with daily or 10 day resolution. Such a data set would be useful in the study of short-term features, such as storm or flood event, assimilation into numerical weather prediction models or for analysis of short-term drought events.

[69] Finally, the final retrieval product will be used in several climatological and case studies. Most of these will focus on assessing the role of soil moisture in climate processes. This involves for instance an investigation of the relationship between ENSO extremes and soil moisture 


\section{KOLASSA ET AL.: SOIL MOISTURE RETRIEVAL METHODOLOGY}

or the influence of the North Atlantic Oscillation on the soil moisture on the Iberian Peninsula. Further studies addressing the influence of soil moisture on convection or the possibility of using soil moisture data for flood prediction will be performed.

[70] Acknowledgments. The research leading to these results has received funding from the European Community's Seventh Framework Programme (FP7 2007-2013) under grant agreement 238366. As such it is part of the GreenCycles II project which "aims to substantially improve the current understanding of the impacts of climate-biogeochemistry feedbacks on the evolution of the Earth system over the next two centuries" (www.greencycles.org). Special thanks are extended to Isabel Trigo and Pedro Viterbo of the Instituto de Meteorologia, Lisbon, Portugal, for providing the HTESSEL soil moisture data as well as very useful support and advice regarding the project. The processing of the ORCHIDEE soil moisture data has been greatly facilitated thanks to the support of Nathalie Bertrand of the Laboratoire de Météorologie Dynamique (LMD), Paris, France.

\section{References}

Aires, F., C. Prigent, and W. B. Rossow (2004), Temporal interpolation of global surface skin temperature diurnal cycle over land under clear and cloudy conditions, J. Geophys. Res, 109, D04313, doi:10.1029/ 2003JD003527.

Aires, F., O. Aznay, C. Prigent, M. Paul, and F. Bernardo (2012), Synergistic multi-wavelength remote sensing versus a posteriori combination of retrieved products: Application for the retrieval of atmospheric profiles using MetOp-A, J. Geophys. Res., 117, D18304, doi:10.1029/ 2011JD017188.

Armstrong, R. L., and M. J. Brodzik (2005), Northern Hemisphere EASEGrid Weekly Snow Cover and Sea Ice Extent Version 3, National Snow and Ice Data Center, Boulder, Colorado.

Balsamo, G., P. Viterbo, A. Beljaars, B. van den Hurk, M. Hirschi, A. Betts, and 1. K. Scipa (2009), A revised hydrology for the ECMWF model: Verification from Field site to terrestrial water storage and impact in the integrated forecast system, J. Hydrol., 10, 623-643.

Balsamo, G., S. Boussetta, P. Lopez, and L. Ferranti (2010), Evaluation of ERA-Interim and ERA-Interim-GPCP-rescaled precipitation over the U.S.A., ERA-Report Series, 5, pp. 10.

Bishop, C. M. (1995), Neural Networks for Pattern Recognition, Oxford University Press, chapter 4.

Cybenko, G. V. (1989), Approximation by superpositions of a sigmoidal function, Mathematics of Control, Signals and Systems, 2, 303-314.

Dorigo, W. A., et al. (2011), The International Soil Moisture Network: A data hosting facility for global in situ soil moisture measurements, Hydrol. Earth Syst. Sci., 15, 1675-1698.

Efroymson, M. A. (1960), Multiple regression analysis, Mathematical Methods for Digital Computers, edited by the A. Ralston, and H.S. Wilf, pp. 191-204, Wiley.

Entekhabi, D., et al. (2010), The Soil Moisture Active Passive (SMAP) Mission, Proceedings of the IEEE, 98(5), 704-716.

Francis, R., et al. (1991), The ERS-1 spacecraft and its payload, ESA Bull., $65,2748$.

Gutman, G. G. (1999), On the use of long-term global data of land reflectances and vegetation indices from the advanced very high resolution radiometer, J. Geophys. Res., 104, 6241-6255.

Horn, B. K. P., and R. J. Woodham (1979), Destriping LANDSAT MSS images using histogram modification, Computer Graphics and Image Processing, 10, 1, 6983.
Jimenez, C., C. Prigent, and F. Aires (2009), Toward an estimation of global land surface heat fluxes from multisatellite observations, J. Geophys. Res., 114, D06305, doi:10.1029/2008JD011392.

Kerr, Y. H., et al. (2010), The SMOS Mission: New tool for monitoring key elements of the global water cycle, Proceedings of the IEEE, 98(5), 666-687.

Liu, Y. Y., R. M. Parinussa, W. A. Dorigo, R. A. M. De Jeu, W. Wagner, A. I. J. M. van Dijk, M. F. McCabe, and J. P. Evans (2011), Developing an improved soil moisture dataset by blending passive and active microwave satellite-based retrievals, Hydrol. Earth Syst. Sci., 15, 425-436.

Njoku, E. G., T. J. Jackson, and V. Lakshmi (2003), Soil moisture retrieval from AMSR-E, IEEE Trans. On Geoscience and Remote Sensing, 41, 215-229.

Owe, M., R. deJeu, and J. Walker (2001), A methodology for surface soil moisture and vegetation optical depth retrieval using the microwave polarization difference index, IEEE Transactions on Geoscience and Remote Sensing, 39, 1643-1654.

Owe, M., R. de Jeu, and T. Holmes (2008), multisensor historical climatology of satellite-derived global land surface moisture, J. Geophys. Res., 113, F01002, doi:10.1029/2007JF000769.

Prigent, C., F. Papa, F. Aires, W. B. Rossow, and E. Matthews (2007), Global inundation dynamics inferred from multiple satellite observations, 1993-2000, J. Geophys. Res., 112, D12107, doi:10.1029/2006JD007847.

Prigent, C., F. Aires, W. B. Rossow, and A. Robock (2005), Sensitivity of satellite microwave and infrared observations to soil moisture at a global scale: Relationship of satellite observations to in situ soil moisture measurements, J. Geophys. Res., 110, D07110, doi:10.1029/ 2004JD005087.

Prigent, C., W. B. Rossow, and E. Matthews (1997), Microwave land surface emissivities estimated from SSM/I observations, J. Geophys. Res., $102,21,867-21,890$.

Rochester, N., J. H. Holland, L. H. Habit, and W. L. Duda (1956), Tests on a cell assembly theory of the action of the brain, using a large digital computer, IRE Transactions on Information Theory, 2(3), 8093, doi:10.1109/ TIT.1956.1056810.

de Rosnay, P., J. Polcher, M. Bruen, and K. Laval (2002), Impact of a physically based soil water flow and soil-plant interaction representation for modeling large-scale land surface processes, J. Geophys. Res., 107 (D11), 4118, 10.1029/2001JD000634

Rossow, W. B., and R. A. Schiffer (1999), Advances in understanding clouds from ISCCP, Bulletin American Meteorological Society, 80, 2261-2287.

Rumelhart, D. E., and Y. Chauvin (1995), Backpropagation: Theory, Architectures, and Applications, Lawrence Erlbaum Associates.

Schmugge, T. J., T. J. Jackson, and H. L. McKim (1980), Survey of methods for soil moisture determination, Water Resources Research, 16(6), 961-979.

Schmugge, T. J., P. E. O’Neill, and J. R. Wang (1986), Passive microwave soil moisture research, IEEE Transactions on Geoscience and Remote Sensing, GE-24(1).

Vinnikov, K. Y., A. Robock, S. Qiu, J. K. Entin, M. Owe, B. J. Choudhury, S. E. Hollinger, and E. G. Njoku (1999), Satellite remote sensing of soil moisture in Illinois, United States, J. Geophys. Res., 104(D4), 4145-4168.

Wagner, W. (1998), Soil moisture retrieval from ERS scatterometer data, Institute for Photogrammetry and Remote Sensing, Vienna University of Technology.

Weedon, G. P., S. Gomes, P. Viterbo, W. J. Shuttleworth, E. Blyth, H. Österle, J. C. Adam, N. Bellouin, O. Boucher, and M. Best (2011), Creation of the WATCH forcing data and its use to assess global and regional reference crop evaporation over land during the twentieth century, J. Hydrometeorology, 12(5), 823-848. 